Check for updates

Cite this: RSC Adv., 2017, 7, 24374

\title{
One-step fabrication of recyclable and robust fluorine/polymer-free superhydrophobic fabrics $\uparrow$
}

\begin{abstract}
Sheng Wang, (D) * Sanding Wu, Jiazhang Zhang and Tao Wang*
Cost-effective, scalable methods to create antiwetting fabric are in high demand for applications ranging from outerwear to water purification and oil-water separation. Conventionally, polymer reagents such as low-surface-tension fluorinated reagents and long alkyl polymers are often incorporated in anti-wetting fabrics to enable water repellency. However, because of their high stabilities and propensity for bioaccumulation, alternative methods that are cost-effective, eco-friendly, and scalable are highly desirable. Herein, we present a simple, environmentally friendly approach to fabricate fluorine-free, recyclable, self-cleaning superhydrophobic coatings. Using only one vacuum heating treatment and without any chemical modification, superhydrophilic $\mathrm{TiO}_{2}$-immobilized polyester fabrics were converted to superhydrophobic fabrics. These fabrics can be applied for oil/water separation, and the adsorbed grease can be photodecomposed quickly by photocatalysis without the use of organic detergents. On the loss of hydrophobicity, the fabric can be heated under vacuum, regenerating the superhydrophobicity.
\end{abstract}

Received 23rd February 2017

Accepted 19th April 2017

DOI: 10.1039/c7ra02276j

rsc.li/rsc-advances

Lotus leaves have a high water contact angle (CA) and low

\section{Introduction}

The design and development of superhydrophobic surfaces with a static water contact angle greater than $150^{\circ}$ and sliding angle below $10^{\circ}$ have garnered tremendous interest in both academic and commercial communities..$^{1-3}$ Over the past two decades, inspired by functional natural biological surfaces, including lotus leaf, butterfly wings, the legs of water striders, gecko feet, and nepenthes leaves, artificial superhydrophobic surfaces have attracted a lot of attention because of their enhanced durability and extended lifespan in practical applications such as self-cleaning, ${ }^{4-6}$ antifouling, ${ }^{7-9}$ antireflection, ${ }^{10-12}$ strong adhesion, ${ }^{13-15}$ and liquid repellency, ${ }^{16-18}$ respectively.

Given the numerous potential applications, various strategies have been proposed to impart superhydrophobic properties to fabrics, for both purely scientific interest and commercial applications. ${ }^{19-22}$ Learning from nature, producing superhydrophobic fabrics has enabled the possibility of applying superhydrophobicity to the field of multifunctional textiles. Despite great advances in scientific community, plenty of limitations still remain, including durability, eco-friendliness, breathability, and so on, which hinder the widespread practical use of superhydrophobic fabrics because of the strict conditions and complicated treatment in most available methods.

Key Laboratory of Advanced Textile Materials and Manufacturing Technology, Zhejiang Sci-Tech University, Hangzhou 310018, China. E-mail: wangsheng571@ hotmail.com; taotao571@hotmail.com

$\dagger$ Electronic supplementary information (ESI) available: Additional XPS, digital photograph of samples, WCA of sample. See DOI: $10.1039 / \mathrm{c} 7 \mathrm{ra02276j}$ sliding angle (SA), and it is now well established that the superhydrophobic wetting state is due to both chemical modification (typically low surface energy constituents) and geometric parameters (typically, the hierarchical roughness) of the solid interface. By controlling the surface morphology of fabrics, novel surface properties can be obtained. Generally, superhydrophobic fabrics are prepared by two different approaches: polymer coating ${ }^{23-26}$ or the integration of inorganic micro/nano-particles with low surface energy fluoro-containing groups or long alkyl groups. ${ }^{27-30} \ln$ both case, the use of organic modification reagents has become a prerequisite for achieving superhydrophobicity. When fluorinated/polymer modified particles have been cast onto various substrates to yield nanoand microarchitectured coatings. Although these coatings exhibit superior water repellency, they (except in rare cases) lack optical clarity and wear resistance. Alternatively, although fluorinated/long alkyl liquid polymers can be grafted onto plain substrates to yield anti-wettability, the drawbacks in this case include the thinness of these monolayers. Increasing the thickness of polymer layer will lead to a significant drop in fabric permeability. Considering that long alkyl polymers or fluorinated reagents are high stability and bioaccumulation. Especially fluorine components are nonbiodegradable and expensive and can easily react with other materials, resulting in environmental contamination that can impede nerve growth in children..$^{31}$ Therefore, fluorine/polymer-free superhydrophobic coatings are highly desirable. Therefore, developing simple, fluorine/polymer-free, low-cost, environmentally friendly, and suitable methods to fabricate superhydrophobic surfaces in large scale is important for industrial application. 
Another challenge in coating on fabric is enhancement of robustness. It is well known that the use of nanoparticles is effective in maintaining the original fabric appearance, flexibility, and permeability after coating. Inorganic micro/nanoparticles are used as the building blocks of the hierarchical surface structure. However, superhydrophobicity and robustness are generally known to be conflicting properties. ${ }^{32}$ As roughness increases, hydrophobicity increases; nevertheless, robustness decreases. As a result, the practical applications of superhydrophobic surfaces have so far been limited on the materials that require robustness. Consequently, realizing both superhydrophobicity and robustness simultaneously by a simple and cost-effective method is still a technical challenge at present.

Herein, we report the one-step generation of superhydrophobic surfaces using inorganic coatings without any organic chemical modification, which converts the superhydrophilic $\mathrm{TiO}_{2} /$ polyester fabric to a robust, recyclable superhydrophobic fabric. The superhydrophobic fabric can be applied to oil/water separation, and grease stains can be decomposed by $\mathrm{Ti}^{3+} / \mathrm{TiO}_{2}$ photocatalysis under UV illumination. When the fabric loses its superhydrophobicity, it can be regenerated by vacuum heating treatment. The method was demonstrated to be used for many combinations of metal oxide nanoparticles and synthetic fabrics, allowing the preparation of a range of synthetic superhydrophobic fabrics. Our method opens a new route to the fabrication of recyclable hydrophobic fabrics without the use of organic reagents.

\section{Experimental}

\section{Materials}

$\mathrm{TiO}_{2}$ nanoparticles (P25) were kindly provided by the Evonik Degussa Specialty Chemicals Company. The average diameter of the P25 nanoparticle was about $21 \mathrm{~nm}$, and the BrunauerEmmett-Teller surface area $\left(S_{\text {BET }}\right)$ was $50 \pm 15 \mathrm{~m}^{2} \mathrm{~g}^{-1}$. Hydrophilic $\alpha-\mathrm{Al}_{2} \mathrm{O}_{3}$ nanoparticles (average diameter: $30 \mathrm{~nm}$ ), $\mathrm{ZnO}$ nanoparticles (average diameter: $30 \pm 10 \mathrm{~nm}$ ), CuO nanoparticles (average diameter: $40 \mathrm{~nm}$ ) and $\mathrm{WO}_{3}$ nanoparticles (average diameter: $50 \mathrm{~nm}$ ) were purchased from Aladdin Co., Ltd. All chemicals were analytical grade reagents and used as received.

Glass slides and commercial polyester fabric (plain weave, $105 \mathrm{~g} \mathrm{~m}^{-2}$, thickness $=120 \mu \mathrm{m}$ ) were used as substrates. Before application as a substrate, the glass slides and polyester fabric were cleaned ultrasonically in water, ethanol, and acetone sequentially for $15 \mathrm{~min}$ each, following drying process in an oven at $60{ }^{\circ} \mathrm{C}$.

\section{Coating procedure}

A certain amount of $\mathrm{TiO}_{2}$ nanoparticles (P25) was dispersed in ethanol, forming a suspension. The suspension was then loaded into an airbrush with a $0.3 \mathrm{~mm}$ nozzle (Iwata Eclipse HPCS) for spray-coating. The operating air was controlled by an airbrush compressor at $20 \mathrm{psi}$, and the distance between the airbrush and the substrate (glass slide or fabric) was kept at 20$25 \mathrm{~cm}$. After spray coating, the sample was placed in a vacuum drying oven at $230{ }^{\circ} \mathrm{C}$ for $3 \mathrm{~h}$ to yield a superhydrophobic surface.

\section{Robustness tests}

We chose knife-scratch, sandpaper-abrasion and laundering durability tests as the robustness tests for the substrates. For the knife-scratch tests, water was first dropped on fabric surface to test the wettability, and, then, a knife (box cutter) was used to scratch the nanoparticles coated surface along a meshy path. Subsequently, water dropping tests were carried out to confirm the robustness of the superhydrophobic surface.

The nanoparticle modified fabric surface was placed facedown on the sandpaper (standard glasspaper, Grit No. 240, from Matador (Germany) Co., Ltd). This surface was longitudinally and transversely $(10 \mathrm{~cm}$ in each direction, or transversely and then longitudinally) abraded by the sandpaper under a weight of $100 \mathrm{~g}$; this process was defined as one cycle. Forty cycles of mechanical abrasion tests were carried out on the fiber samples, and the water contact angles were measured after each abrasion test cycle. In the water droplet traveling tests, water droplets were guided by a needle across the treated surface after the 10th, 20th, and 30th, abrasion tests, respectively.

The laundering durability was tested by a standard procedure according to AATCC Test Method 61-2003 Test No. 1A. Samples were washed using a laundering machine (SW-12 E, Fang Yuan, China) at $40{ }^{\circ} \mathrm{C}$ in the presence of the nanoparticle modified fabric with the existence of $0.37 \mathrm{wt} \%$ soap powder. One laundering cycle (45 $\mathrm{min}$ ) is approximate to five instances of commercial laundering. The laundering fabric were rinsed with abundant water to remove the residual detergent and dried at $80^{\circ} \mathrm{C}$.

\section{Water vapor permeability test}

Water vapor permeability was evaluated using the ASTM E-96 (open cup test) method. The test fabric sample was placed tightly over a shallow dish containing distilled water. The weight loss of the test assembly over $24 \mathrm{~h}$ was measured, and the vapor transmission rate ( $\mathrm{g}$ per $\mathrm{m}^{2}$ per day) was calculated as water vapor permeability.

\section{Separation of the oil phase from an oil/water mixture}

To investigate the oil/water separation, we fixed the $\mathrm{P} 25-\mathrm{VH} /$ polyester fabric between a transparent glass feeder and a suction flask using a clip, and the joints were sealed using vacuum grease. The oil/water $(\mathrm{v} / \mathrm{v}=1: 1)$ mixture was poured onto the fabric and separated by gravity. Seven types of oil/water mixtures, including toluene/water, dichloromethane/water, gasoline/water, vegetable oil/water, petroleum ether/water, chloroform/water, and hexadecane/water, were separated by the $\mathrm{P} 25-\mathrm{VH} /$ polyester fabric. The separation efficiency of each mixture was then calculated.

\section{Stability measurement}

The P25-VH/polyester fabric was prepared by vacuum heating treatment. Then, the modified fabric was exposed to light (indoor lamp illumination) at room temperature $\left(25 \pm 2{ }^{\circ} \mathrm{C}\right)$ 
with a relative humidity of $60 \pm 2 \%$. The surface wettability and XPS spectra of the exposed sample were measured each week.

\section{Characterization}

Scanning electron microscopy (SEM) images were taken using a field-emission scanning electron microscopy (FESEM, ZEISS VLTRA-55, $10 \mathrm{kV}$ ). Energy dispersive X-ray analysis (EDS, IncaEnergy-200) was used to investigate the sample compositions. X-ray diffraction (XRD) patterns were obtained on a $\mathrm{D} /$ MAX-RB X-ray diffractometer (D/Max-2550pc) using $\mathrm{Cu}-\mathrm{K} \alpha$ radiation at a scan rate $(2 \theta)$ of $0.05^{\circ} \mathrm{s}^{-1}$, and the patterns were used to determine the phase structure of the obtained samples. The accelerating voltage and the applied current were $15 \mathrm{kV}$ and $20 \mathrm{~mA}$, respectively. The elemental analysis of the samples was carried out by X-ray photoelectron spectroscopy (XPS; Thermo Scientific K-Alpha) was used, with a monochromatized $\mathrm{Al} \mathrm{K} \alpha \mathrm{X}$ ray source operated at $12 \mathrm{kV}$ and $20 \mathrm{~mA}$. Photoelectrons were collected at a takeoff angle of $90^{\circ}$ relative to the sample surface. Wide-scan survey spectra were acquired using an analyzer pass energy of $100 \mathrm{eV}$ and a step size of $1 \mathrm{eV}$. All binding energy values were calculated relative to the $C(1 \mathrm{~s})$ photoelectron at $285 \mathrm{eV}$. Fourier-transform infrared (FTIR) spectra were recorded using a Nicolet 5700 FTIR spectrometer. The resolution was 4 $\mathrm{cm}^{-1}$, and the average result of 60 automatic scans from 4000 to $650 \mathrm{~cm}^{-1}$ was output as the test result.

The static contact angles (CA) for water on the coatings was measured by the sessile drop method using a DSA-100 optical contact-angle meter (Kruss Company, Ltd. Germany) using liquid droplets of $5 \mu \mathrm{L}$ water in volume. The sliding angles (SA) were measured by a tilting stage method (BOSCH DWM40L). For each sample, the CAs and SAs were measured at 5 different positions, and the reported values are the average of these measurements.

\section{Results and discussion}

The superhydrophobic surfaces were fabricated following the procedure shown in Scheme 1. A suspension of $\mathrm{TiO}_{2}$ (P25) nanoparticles was sprayed onto the substrates evenly (either a glass slide or polyester fabric) by a nozzle spray gun. Then, the substrates were placed in a vacuum drying oven for $3 \mathrm{~h}$ at $230^{\circ} \mathrm{C}$ to prepare the superhydrophobic surface.
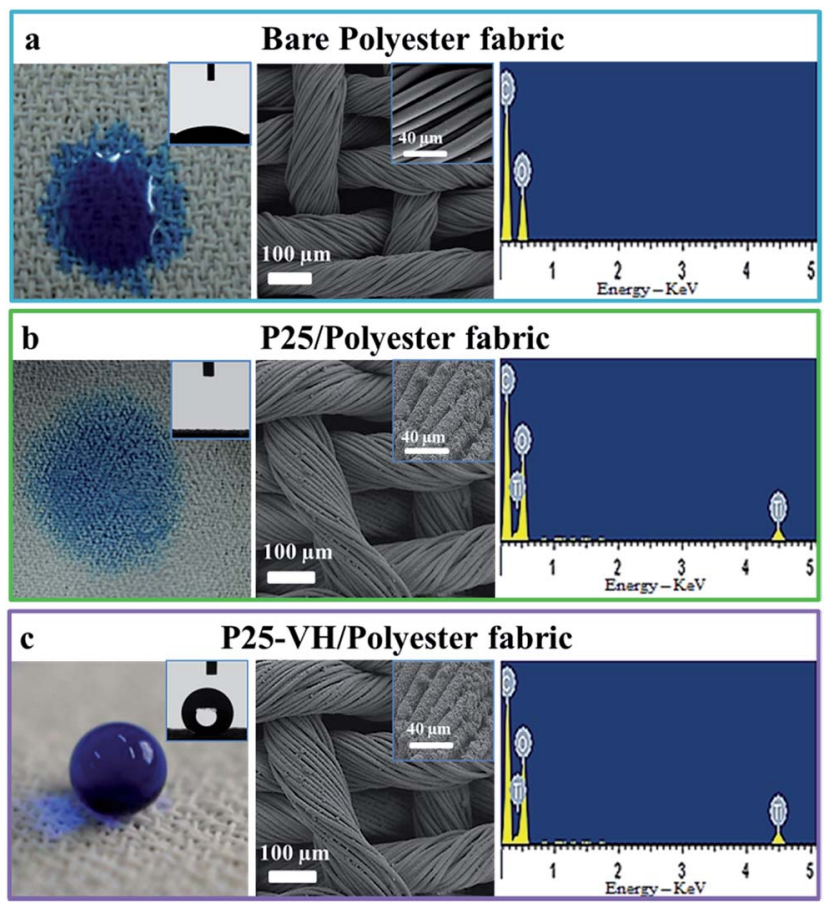

Fig. 1 Digital photographs, SEM images, CA, and EDX measurements of different polyester fabric samples: (a) bare polyester fabric; (b) polyester fabric with pristine P25 coating; and (c) superhydrophobic $\mathrm{P} 25-\mathrm{VH} /$ polyester fabric after vacuum heating treatment.

Scanning electron microscopy (SEM) analyses were performed to examine the surface morphology of the as-prepared samples. Fig. 1a and b show the morphologies of the polyester fabric before and after the coating treatment. From the images, we can see that the cloth has a microstructure comprising compact bundle fibers, which is an artificial pseudo-two-dimensional network. The fasciculus, which has a diameter of approximately $100 \mu \mathrm{m}$, consists of a bundle of fibers with an average diameter of around $12 \mu \mathrm{m}$. After loading with the P25 titania coating, the SEM images revealed that the angular P25 nanoparticles, which have diameters of 20 to $40 \mathrm{~nm}$, were aggregated randomly on the surface of the polyester fibers. The untreated pristine fabric was hydrophilic. Generally, increased surface roughness enhances the surface wettability, so changes in the surface roughness allow the

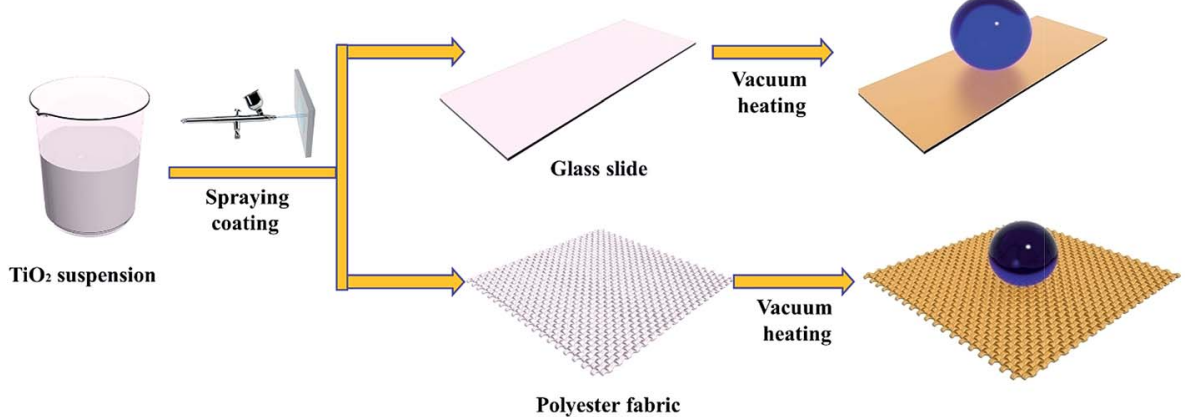

Scheme 1 Schematic illustration of the preparation of superhydrophobic $\mathrm{TiO}_{2}$ coating. 
original hydrophilic or hydrophobic surfaces to become either more hydrophilic or hydrophobic, respectively. The P25 coating is superhydrophilic, as shown by its CA of $0^{\circ}$. The surface can be easily wetted with water for two reasons: first, the P25 nanoparticles are inherently hydrophilic and, secondly, the rough surface structure enhanced the hydrophilic property of the glass surface. After vacuum storage at $230{ }^{\circ} \mathrm{C}$ for $3 \mathrm{~h}$, no obvious morphology changes were observed. However, Fig. 1c clearly shows a nearly spherical water droplet that remained on the treated fabric surface. The CA of the polyester fabric loaded with vacuum treated P25 (denoted P25-VH) had a water contact angle of $156 \pm 1^{\circ}$ and a sliding angle of $4.5 \pm 0.1^{\circ}$. The P25-VH loaded glass slide also showed a dramatic change in wettability.

To understand the changes in wettability, we used a glass slide loaded with $\mathrm{P} 25-\mathrm{VH}$ as a representative sample to test the superhydrophobic coatings (Fig. 2). The SEM images reveal that the bare glass slide had a homogeneous and smooth surface, and energy-dispersive X-ray spectroscopy (EDX) detected signals corresponding to $\mathrm{Na}, \mathrm{Ca}, \mathrm{Si}$, and $\mathrm{O}$. The bare glass surface was hydrophilic due to the presence of hydroxyl groups, and the CA of water was $43 \pm 1^{\circ}$. The P25-loaded glass slide was superhydrophilic, with a $\mathrm{CA}$ of $0^{\circ}$. After vacuum heating, the coating became superhydrophobic $\left(\mathrm{CA}=163 \pm 1^{\circ}\right.$ and SA of $\left.2.5 \pm 0.1^{\circ}\right)$. From EDX analysis, we found that there was no difference between samples $\mathrm{c}$ and d, both of which contain $\mathrm{Ti}, \mathrm{Na}, \mathrm{Ca}, \mathrm{Si}$, and $\mathrm{O}$, indicating that the vacuum heating process did not introduce new elements to the surface.

Notably, on the collection of the vacuum heated samples, we noticed a distinct color change, from white (P25) to light brown
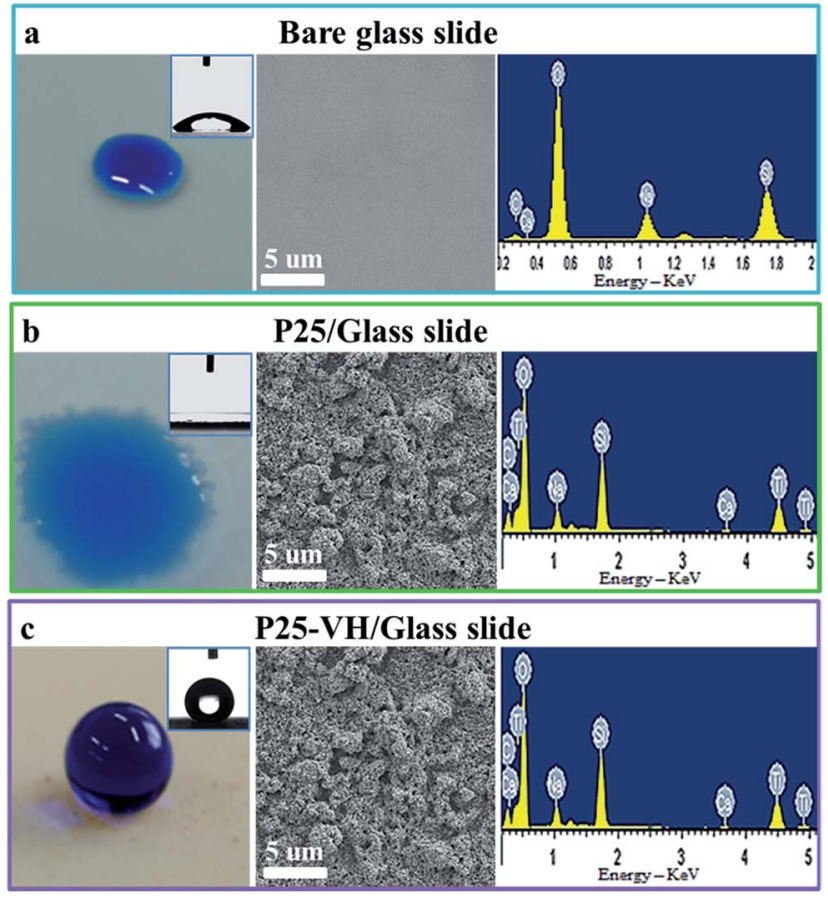

Fig. 2 Digital photographs, SEM images, CA, and EDX measurements of different glass slide samples: (a) bare glass slide; (b) glass slide with pristine P25 coating; and (c) superhydrophobic P25-VH/glass slide after vacuum heating treatment.
(P25-VH) (Fig. 3a). On immersion in water, the collected nanoparticles floated on the surface, indicating that wettability of the nanoparticles had changed from hydrophilic to hydrophobic.

The X-ray diffraction (XRD) patterns of the samples showed that no obvious anatase-to-rutile phase transformation occurred during vacuum heating, suggesting that $\mathrm{TiO}_{2}$ remains in the P-25 phase throughout the preparation procedure (Fig. 4).

Fig. S1 (ESI, S1†) shows the X-ray photoelectron spectroscopy (XPS) survey spectra results of P25 and P25-VH nanoparticles surface before and after the vacuum heating procedure. The spectra clearly indicate the presence of Ti and O. Fig. $3 \mathrm{~b}$ shows the XPS high-resolution Ti $2 \mathrm{p}$ spectrum with and without vacuum heating treatment. We found that the Ti $2 \mathrm{p}_{3 / 2}$ peak of the $\mathrm{P} 25-\mathrm{VH}$ sample was broader and at a lower binding energy $(458.41 \mathrm{eV})$ than that of P25. As proposed by Wang et al., ${ }^{33,34}$ the broadening of the Ti $2 \mathrm{p}_{3 / 2}$ peak at low binding energies can be attributed to the presence of $\mathrm{Ti}^{3+}$. The deconvolution of the $\mathrm{Ti}$ $2 \mathrm{p}$ spectrum allowed the assignment of the signals centered at 458.68 and $457.9 \mathrm{eV}$ to $\mathrm{Ti}^{4+}$ and $\mathrm{Ti}^{3+}$, respectively. ${ }^{35,36}$

Electron paramagnetic resonance (EPR) spectra of the P25VH samples show a very strong EPR signal at $g=1.998$, while no signal was seen in the spectrum of pure P25 (Fig. 3c). Paramagnetic $\mathrm{Ti}^{3+}$ has a $g$-value of 1.94-1.99; oxygen vacancies $\left(\mathrm{O}_{\mathrm{v}}{ }^{*}\right)$ at $g=2.004 .^{37,38}$ The EPR signal in the P25-VH sample is located at $g=1.998$, indicating the presence of both $\mathrm{Ti}^{3+}$ and $\mathrm{O}_{\mathrm{v}}{ }^{\cdot 35,36}$ Many studies have indicated that $\mathrm{Ti}^{3+}$ and $\mathrm{O}_{\mathrm{v}}{ }^{\circ}$ are both defects on the surface of $\mathrm{TiO}_{2}$ nanoparticles and are produced at elevated temperatures. Under oxygen-rich conditions, oxygen vacancies are the favored defect type. Under oxygen-poor conditions, both defect types are stabilized, with $\mathrm{Ti}^{3+}$ predicted to be favored. ${ }^{39,40}$ Therefore, after vacuum heating, the primary defect species is believed to be $\mathrm{Ti}^{3+}$. Fourier-transform infrared (FTIR) spectra of the samples were measured, and these are shown in Fig. 3e. The signals corresponding to adsorbed water on $\mathrm{TiO}_{2}$ with maxima at 3400 , and $1638 \mathrm{~cm}^{-1}$, and these were assigned to the $\mathrm{O}-\mathrm{H}$ stretching and $\mathrm{H}-\mathrm{O}-\mathrm{H}$ bending vibrations. After vacuum heating, the band at 3400 and $1638 \mathrm{~cm}^{-1}$ almost disappeared. This result agrees well with the previous report that, under vacuum heating conditions $(>150$ ${ }^{\circ} \mathrm{C}$ ), the physically-adsorbed water is completely removed. ${ }^{41}$

$\mathrm{TiO}_{2}$ is a well-known photocatalyst, and, to better understand its various functions, the structure and behavior of adsorbed water have been extensively investigated using several techniques. As described by Takeuchi et al., when both $\mathrm{Ti}^{3+}$ and oxygen vacancies are present on the $\mathrm{TiO}_{2}$ surface, the surface is negatively charged, resulting in the poor surface adsorption of water molecules because of repulsive effects (Scheme 2). ${ }^{42,43}$ Therefore, the vacuum heating treatment removes water molecules absorbed on the P25 surface, producing $\mathrm{Ti}^{3+}$ defects and oxygen vacancies simultaneously, which consequently changes the surface properties.

Interestingly, we found that the superhydrophobic P25-VH was very stable. Even after storage at room temperature in the presence of light (room lighting), $\mathrm{O}_{2}$, and humidity (60\% humidity) for one month, the CA and SA only showed slight changes. Notably, the EPR spectra do not indicate the presence of $\mathrm{Ti}^{3+}$ on the surface of the sample (as surface $\mathrm{Ti}^{3+}$ is highly 

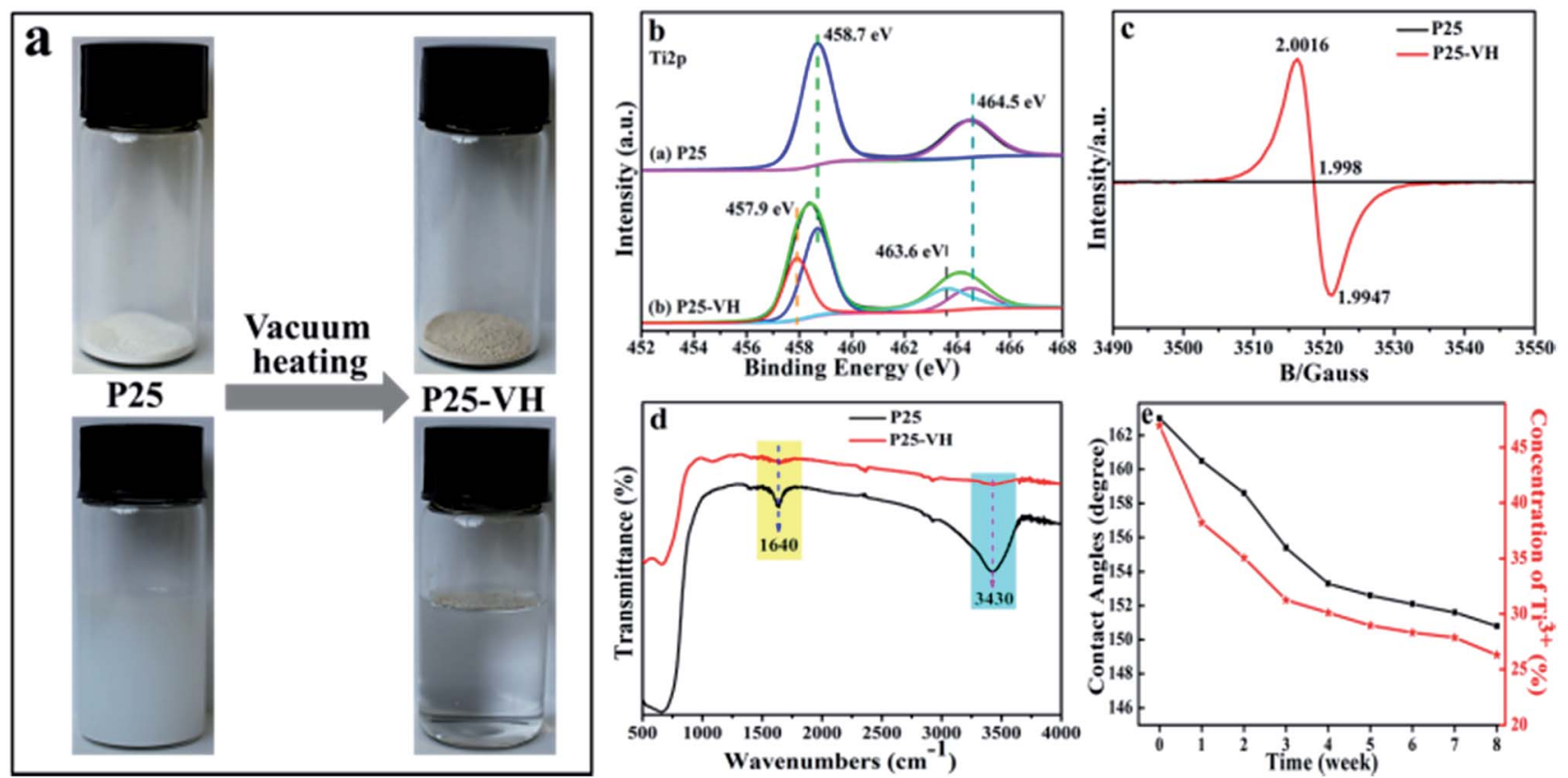

Fig. 3 (a) Digital photographs of collected P25 and P25-VH samples and wettability tests; bare polyester fabric; (b) XPS core level spectra of the Ti $2 \mathrm{p}$ peaks; (c) EPR spectra for P25 and P25-VH; (d) FTIR spectra for P25 and P25-VH; and (e) the relationship between retention time and concentration of $\mathrm{Ti}^{3+}$ and $\mathrm{CA}$.

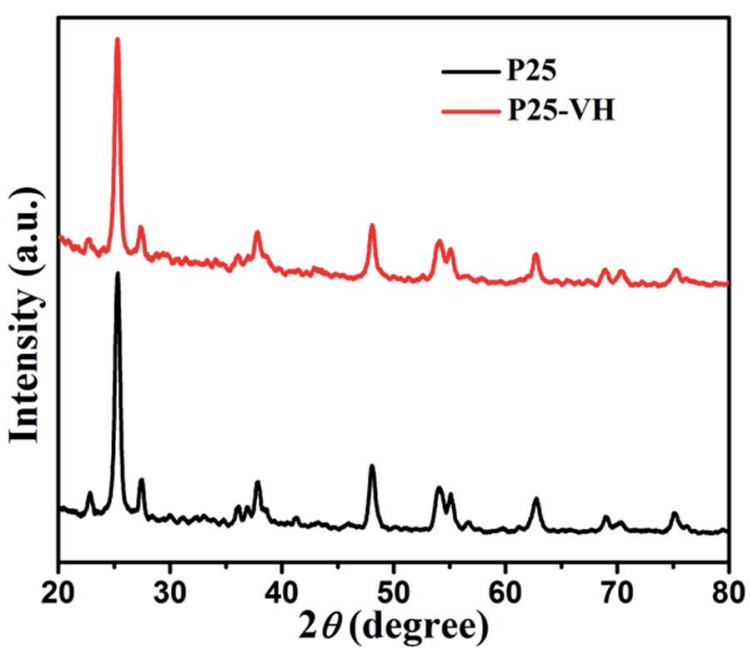

Fig. 4 XRD patterns of P25 and P25-VH samples.

unstable in air). Therefore both the XPS and EPR results suggest that the $\mathrm{Ti}^{3+}$ is located in the bulk rather than the surface, and this is crucial for the stability of the sample. ${ }^{44}$ The relationship between the retention time and the concentration of $\mathrm{Ti}^{3+}$ and CA was investigated to evaluate the decay of the defects (Fig. 3d). After vacuum heating treatment, a quantitative XPS analysis was carried out to determine the $\mathrm{Ti}^{3+} /\left(\mathrm{Ti}^{3+}+\mathrm{Ti}^{4+}\right)$ atomic ratio, which was found to be about $47 \%$; that is, nearly half of the titanium species in $\mathrm{TiO}_{2}: \mathrm{Ti}^{3+}$ are present as $\mathrm{Ti}^{3+}$. At this high $\mathrm{Ti}^{3+}$ concentration, the sample was superhydrophobic. To investigate the stability of the samples, we stored a hydrophobic $\mathrm{P} 25-\mathrm{VH} / \mathrm{glass}$ slide at $25 \pm 2{ }^{\circ} \mathrm{C}$ and $60 \pm 2 \%$ humidity. With increasing storage time, the concentration of $\mathrm{Ti}^{3+}$ decreased gradually, as did the CA of the water droplets. However, the hydrophilic recovery was very slow (longer than two month), and the hydrophilicity was only partially recovered.

On the other hand, for a superhydrophobic surface using inorganic nanoparticles to provide surface roughness, superhydrophobicity and robustness are generally conflicting properties. That is, as the roughness increases, the hydrophobicity increases and the robustness decreases..$^{45}$ Conventional strategies used to enhance the robustness of the material typically involve chemical bonding between the superhydrophobic coating and the substrate or cross-linking the coating layers by using organic fluorinated compounds or long chain alkyl groups. ${ }^{45,46}$ Another advantage of our method is the lack of use of organic adhesives, and the nanoparticles were firmly loaded on the polyester fabric.

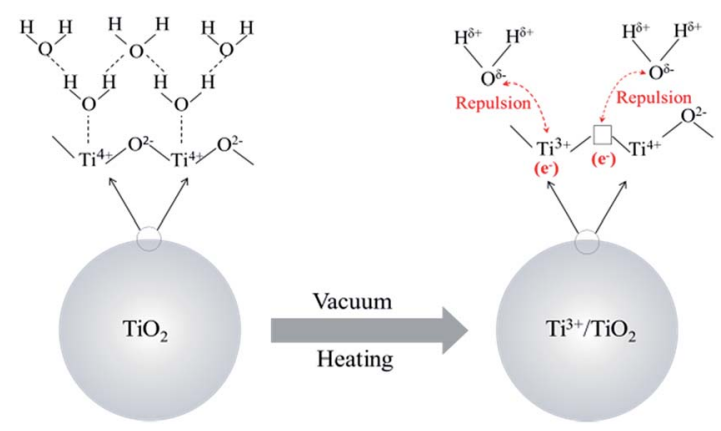

Scheme 2 Interaction model of absorbed water on $\mathrm{TiO}_{2}$ before and after vacuum heating treatment. 
For common polyester fabrics, the softening temperatures range from 230 to $240{ }^{\circ} \mathrm{C}$. When the $\mathrm{P} 25$-loaded polyester fabric was heated to $230{ }^{\circ} \mathrm{C}$ at a constant pressure in the presence of $\mathrm{O}_{2}$, the fibers lost their original morphology, forming a yellow film after $3 \mathrm{~h}$, indicating that melting and ageing occurred during the heating process (ESI, S2 $\dagger$ ). Both SEM images and FTIR results showed that the vacuum heating treatment is beneficial, protecting the fabric from such thermal-oxidative ageing behavior and maintaining the fiber morphology without any chemical changes (Fig. 5). Therefore, it is considered that at the softening temperature, the polymer material becomes soft and sticky, which causes nanoparticles depress slightly into the surface of the softening polymer. Because of the large specific surface area of the nanoparticles, the contact area between the nanoparticles and polymer should be large. When the temperature gradually decreases, with the solidification of polymer, the nanoparticles are embedded into bulk polymer firmly. At the same time, in the absence of $\mathrm{O}_{2}$, thermal-oxidative aging could not occur. Thus, the morphology and chemical structure of the fabric material were maintained.

Knife-scratch and sandpaper-abrasion tests were chosen to test the robustness of the coated fabric. As shown in Fig. 6a and Movie S1, $\uparrow$ after the knife-scratch tests, the surface retained its superhydrophobicity. Consequently, due to the good compatibility of the superhydrophobic nanoparticles and the fabric, the superhydrophobic surfaces were highly robust. To investigate the robustness further, we conducted a sandpaper-abrasion test using a polyester fabric sample (Fig. 6b). The fabric sample was placed face-down on a piece of sandpaper under a weight of $100 \mathrm{~g}$ and moved longitudinally by $10 \mathrm{~cm}$ along a ruler. Subsequently, the sample was rotated by $90^{\circ}$ in situ and then moved transversely by $10 \mathrm{~cm}$ along the ruler. One transverse abrasion. After 30 cycles of the sandpaper-abrasion obvious damage to the fabric, aside from the fracture of test, SEM images were taken, and these show that there was no abrasion cycle was defined as one longitudinal and one individual fibers (Fig. 6c). Although the static water contact angles showed a slight decrease after 30 abrasion cycles, falling slightly to $147^{\circ}$, the superhydrophobic fabric surface showed excellent robustness (Movie S2 $\dagger$ ).

Fig. $6 \mathrm{~d}$ shows the static water contact angles after 30 cycles of sandpaper-abrasion tests on the fiber samples prepared at

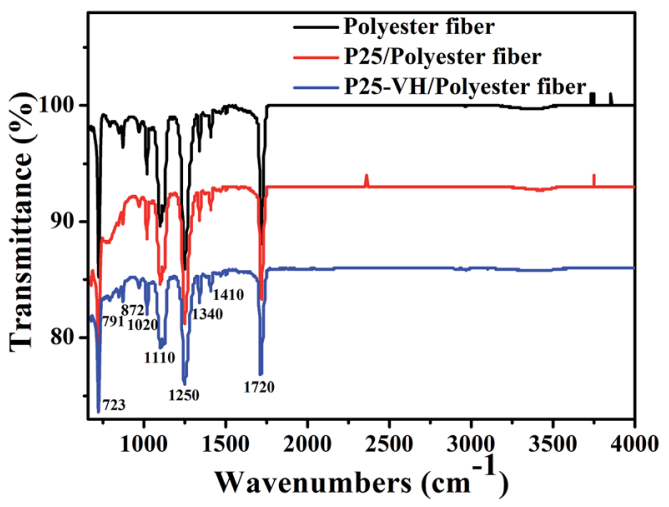

Fig. 5 ATR-FTIR spectra of the different fabric samples.
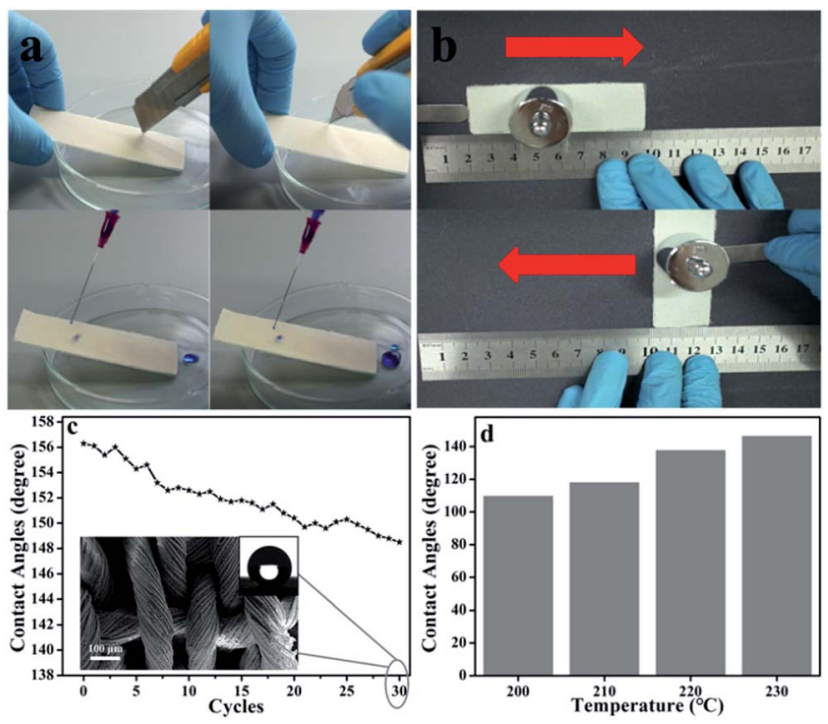

Fig. 6 (a) Knife-scratch tests on the superhydrophobic surfaces of $\mathrm{P} 25-\mathrm{VH} /$ polyester fabric; and (b) sandpaper-abrasion tests after one cycle of the sandpaper-abrasion test, respectively; (c) plot of mechanical abrasion cycles and CAs after each abrasion test (inset: SEM image of fabric surface after sandpaper-abrasion tests for 30 cycles); (d) the CAs of water on 30 cycles of sandpaper-abrasion tested fabric that were treated at different vacuum heating temperatures.

different heating temperatures. As the treatment temperature was increased, the robustness of the superhydrophobic fiber increased; that is, at vacuum heating temperatures of 200, 210, 220 , and $230^{\circ} \mathrm{C}$, the CAs of water on 30 cycles of sandpaperabrasion tested fabric were $110,118,138$, and $147^{\circ}$, respectively. Thus, concerning hydrophobicity, the sample prepared at $230{ }^{\circ} \mathrm{C}$ showed the best performance.

Laundering is an important cause of degradation to superhydrophobic coatings during their practical uses. Fabric during washing undergoes mechanical stresses in addition to chemical agents. The synergistic effect of chemical and physical actions accelerates the detachment of coating layer on fiber substrate. The loading fabric was further evaluated by a laundering test (ESI, S3 $\dagger$ ). After 20 laundering cycles, the CA of the loading fabric decreased from $156^{\circ}$ to $0^{\circ}$, but the loading ratio of $\mathrm{TiO}_{2}$ nanoparticles still remained at $1.94 \%$ (the original loading ratio is $2.8 \%$ ), indicating the excellent mechanical stability of the $\mathrm{P} 25-\mathrm{VH} /$ polyester fabric.

The balance between new functionalities and the desired fabric natures is important for finished fabric products. Therefore, water vapor transmission rates for $\mathrm{P} 25-\mathrm{VH} /$ polyester fabrics were tested at $25{ }^{\circ} \mathrm{C}$ and $50 \% \mathrm{RH}$ for 1 day (ESI, S4 $\dagger$ ). There was no obvious change during the coating treatment, which is satisfactory for practical use.

As a proof of concept, an oil/water separation experiment using the $\mathrm{P} 25-\mathrm{VH} /$ polyester fabric was performed using a simple setup. Fig. 7a shows the setup for separating a dibromoethane (red color)/water (blue color) mixture (Movie S3†). Due to the superhydrophobicity and superoleophilicity of the fabric, water was retained above the fabric, while the oil phase quickly spread out, permeating the fabric quickly and falling into the beaker 

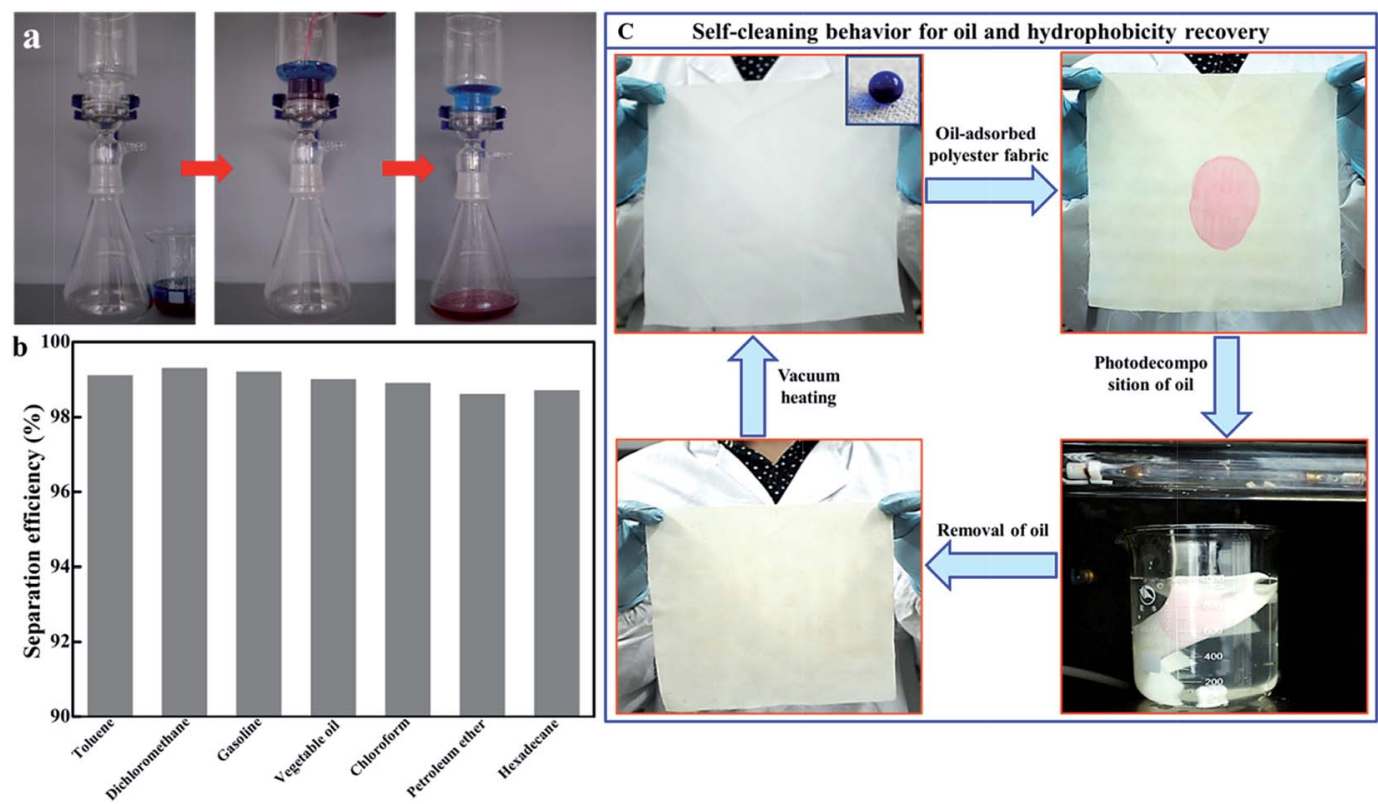

Fig. 7 (a) Selective oil/water separation using the P25-VH/polyester fabric; (b) variations in oil separation efficiency of different types of oil/water mixtures; (c) self-cleaning behavior of $\mathrm{P} 25-\mathrm{VH} /$ polyester fabric and hydrophobicity recovery.

flask below. Fig. 7b shows the variations in oil separation efficiency of different types of oil/water mixtures. The as-prepared $\mathrm{P} 25-\mathrm{VH} /$ polyester fabric is very effective at separating different types of oil/water mixtures, with separation efficiencies (greater than 98\%), demonstrating that this fabric is an excellent candidate for the treatment of oil polluted water.

For the oil/water separation measurements, a square piece of the $\mathrm{P} 25-\mathrm{VH} /$ polyester fabric measuring $25 \mathrm{~cm} \times 25 \mathrm{~cm}$ was

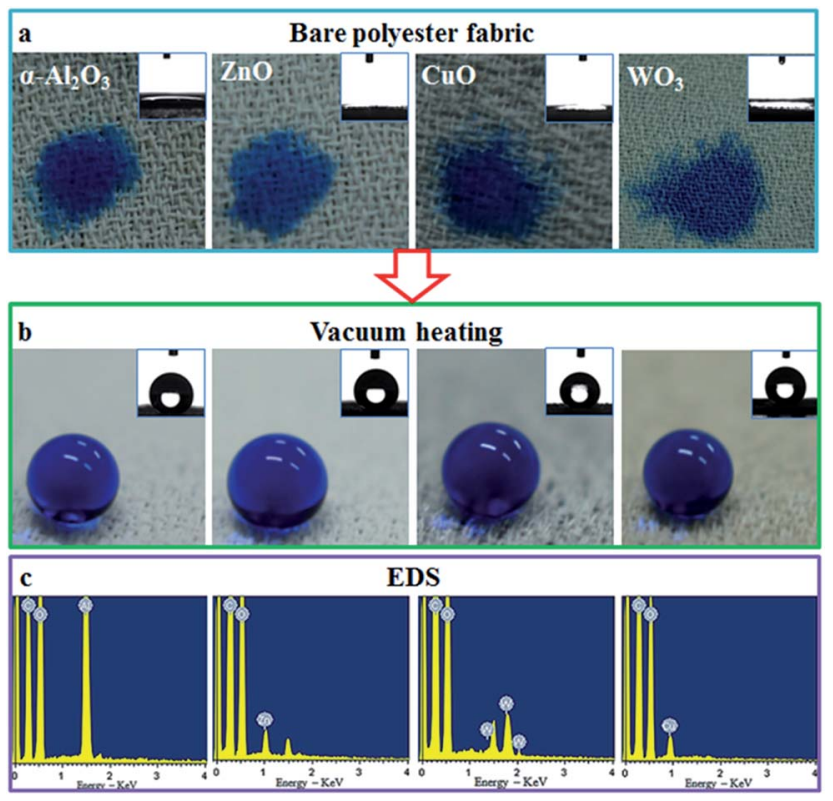

Fig. 8 Vacuum heating treatment of different metal oxide nanoparticle-loaded polyester fabrics and the CAs of water droplets on the samples, respectively. prepared, as shown in Fig. 4c. Generally, when continuously applied for oil/water separation, a small amount of oil will be absorbed into the fabric, forming grease spots (dichloromethane, red color). Consequently, we tested using the photocatalytic ability of $\mathrm{TiO}_{2}$ for the degradation of organic compounds. On light exposure, although the $\mathrm{P} 25-\mathrm{VH} /$ polyester fabric lost its superhydrophobicity, the fabric acted as a selfcleaning surface. Without using any synthetic detergents to remove the organic compounds, the grease spots were photodecomposed (after approximately $6 \mathrm{~h}$ ) using UV light. However, after continuous UV irradiation, the fabric gradually lost its superhydrophobicity and became superhydrophilic, with the water droplets spreading out immediately on the fabric surface. This arises mainly because of the transformation of $\mathrm{Ti}^{3+} / \mathrm{TiO}_{2}$ to $\mathrm{TiO}_{2}$. However, we found that a subsequent vacuum heating treatment allowed the superhydrophilic $\mathrm{TiO}_{2} /$ polyester fabric to recover its superhydrophobic state.

Furthermore, in addition to $\mathrm{TiO}_{2}$, our vacuum heating method can also be used with other metal oxides such as $\alpha$ $\mathrm{Al}_{2} \mathrm{O}_{3}, \mathrm{ZnO}, \mathrm{CuO}$, and $\mathrm{WO}_{3}$ nanoparticles, which all yield fabrics with excellent superhydrophobicity (Fig. 8). However, when the particle size was increased to the micro-scale, superhydrophobic performance was not achieved (e.g., 1 to $2 \mu \mathrm{m}$ sized particles of $\mathrm{Al}_{2} \mathrm{O}_{3}$, and $\mathrm{ZnO}$ failed to achieve superhydrophobicity). Therefore, the superhydrophobicity is related to the size of the metal oxide nanoparticles. Finally, we changed the polyester fabric for nylon 6 (softening at $180^{\circ} \mathrm{C}$ ), and this fabric also showed excellent superhydrophobicity (ESI, S5†).

\section{Conclusions}

In summary, using only one vacuum heating treatment and without any chemical modification, superhydrophilic $\mathrm{TiO}_{2}{ }^{-}$ 
immobilized polyester fabrics were converted to superhydrophobic fabrics. These fabrics can be applied for oil/water separation, and the adsorbed grease can be photodecomposed quickly by photocatalysis without the use of organic detergents. On the loss of hydrophobicity, the fabric can be heated under vacuum, regenerating the superhydrophobicity. Considering the applications of our samples and the simplicity of our vacuum heating method to prepare different kinds of metal oxides on synthetic fabrics, we anticipate that this scalable, fluorine-free method will allow the preparation of many new hydrophobic fabrics. We hope that these coatings can be applied readily to a diverse range of synthetic fabric substrates in the near future.

\section{Acknowledgements}

We thank the National Natural Science Foundation of China (No. 51471153 and 51372227), and 521 Talent Project of Zhejiang Sci-Tech University for providing financial support.

\section{References}

1 A. K. Kota, G. Kwon, W. Choi, J. M. Mabry and A. Tuteja, Nat. Commun., 2012, 3, 1025-1032.

2 X. Yao, Y. Song and L. Jiang, Adv. Mater., 2011, 23, 719734.

3 Y. S. Joung and C. R. Buie, ACS Appl. Mater. Interfaces, 2015, 7, 20100-20110.

4 B. Bhushan and Y. C Jung, Prog. Mater. Sci., 2011, 56, 1-108.

5 Y. Lai, Y. Tang and J. Gong, J. Mater. Chem., 2012, 22, 74207426.

6 C. Kapridaki and P. Maravelaki-Kalaitzaki, Prog. Org. Coat., 2013, 76, 400-410.

7 W. Van Zoelen, H. G. Buss and N. C. Ellebracht, ACS Macro Lett., 2014, 3, 364-368.

8 X. Ding, C. Yang and T. P. Lim, Biomaterials, 2012, 33, 65936603.

9 S. Nishigochi, T. Ishigami and T. Maruyama, Ind. Eng. Chem. Res., 2014, 53, 2491-2497.

10 J. H. Shin, K. S. Han and H. Lee, Prog. Photovoltaics, 2011, 19, 339-344.

11 K. Y. Lai, Y. R. Lin and H. P. Wang, CrystEngComm, 2011, 13, 1014-1017.

12 Y. R. Lin, K. Y. Lai and H. P. Wang, Nanoscale, 2010, 2, 27652768.

13 J. H. Jiang, L. P. Zhu and X. L. Li, J. Membr. Sci., 2010, 364, 194-202.

14 W. Zhao, L. Wang and Q. Xue, J. Phys. Chem. C, 2010, 114, 11509-11514.

15 Y. Lai, X. Gao and H. Zhuang, Adv. Mater., 2009, 21, 37993803.

16 M. Hikita, K. Tanaka and T. Nakamura, Langmuir, 2005, 21, 7299-7302.

17 A. K. Epstein, B. Pokroy and A. Seminara, Proc. Natl. Acad. Sci. U. S. A., 2011, 108, 995-1000.

18 M. K. Tiwari, I. S. Bayer and G. M. Jursich, ACS Appl. Mater. Interfaces, 2010, 2, 1114-1119.
19 H. Zhou, Y. Zhao, H. Wang and T. Lin, Adv. Mater. Interfaces, 2016, 3, 1600402-1600421.

$20 \mathrm{H}$. Teisala, M. Tuominen and J. Kuusipalo, Adv. Mater. Interfaces, 2014, 1, 1300026-1300045.

21 M. Wolfs, T. Darmanin and F. Guittard, Polym. Rev., 2013, 53, 460-505.

22 B. Su, Y. Tian and L. Jiang, J. Am. Chem. Soc., 2016, 138, 17271748.

23 M. Fortuny, C. Graillat and T. F. McKenna, Ind. Eng. Chem. Res., 2004, 43, 7210-7219.

24 S. Michielsen and H. J. Lee, Langmuir, 2007, 23, 6004-6010. 25 C. Ruan, K. Ai, X. Li and L. Lu, Angew. Chem., Int. Ed., 2014, 53, 5556-5560.

26 H. Zhou, H. Wang, H. Niu, J. Fang, Y. Zhao and T. Lin, Adv. Mater. Interfaces, 2015, 2, 1400559-1400566.

27 B. Xu and Z. Cai, Appl. Surf. Sci., 2008, 254, 5899-5904.

28 S. Li, J. Huang, M. Ge, C. Cao, S. Deng, S. Zhang, G. Chen, K. Zhang, S. S. Al-Deyab and Y. Lai, Adv. Mater. Interfaces, 2015, 2, 1500220-1500230.

29 H. Wang, J. Fang, T. Cheng, J. Ding, L. Qu, L. Dai, X. Wang and T. Lin, Chem. Commun., 2008, 877-879.

30 M. E. Yazdanshenas and M. Shateri-Khalilabad, Ind. Eng. Chem. Res., 2013, 52, 12846-12854.

31 A. L. Choi, G. Sun, Y. Zhang and P. Grandjean, Environ. Health Perspect., 2012, 120, 1362-1368.

32 B. Chen, J. Qiu, E. Sakai, N. Kanazawa, R. Liang and H. Fang, ACS Appl. Mater. Interfaces, 2016, 8, 17659-17667.

33 L. Q. Wang, K. F. Ferris, P. X. Skiba, A. N. Shultz, D. R. Baer and M. H. Engelhard, Surf. Sci., 1999, 440, 60-68.

34 J.-Y. Zheng, S.-H. Bao, Y. Guo and P. Jin, ACS Appl. Mater. Interfaces, 2014, 6, 1351-1355.

35 M. Xing, J. Zhang, F. Chen and B. Tian, Chem. Commun., 2011, 47, 4947-4949.

36 D. Courcot, L. Gengembre, M. Guelton, Y. Barbaux and B. Grzybowska, J. Chem. Soc., Faraday Trans., 1994, 90, 895-898.

37 C. Conesa and J. Soria, J. Phys. Chem., 1982, 86, 1392-1395. 38 I. Nakamura, N. Negishi, S. Kutsuna, T. Ihara, S. Sugihara and K. Takeuchi, J. Mol. Catal. A: Chem., 2000, 161, 205-212.

39 A. Pan, M. Yang, X. Fu, N. Zhang and Y. Xu, Nanoscale, 2013, 5, 3601-3614.

40 A. Naldoni, M. Allieta, S. Santangelo, M. Marelli, F. Fabbri, S. Cappelli, C. L. Bianchi, R. Psaro and V. Dal Santo, J. Am. Chem. Soc., 2012, 134, 7600-7603.

41 H. Lin, J. Long, Q. Gu, W. Zhang, R. Ruan, Z. Li and X. Wang, Phys. Chem. Chem. Phys., 2012, 14, 9468-9474.

42 L.-M. Liu, P. Crawford and P. Hu, Prog. Surf. Sci., 2009, 84, 155-176.

43 M. Takeuchi, G. Martra, S. Coluccia and M. Anpo, J. Phys. Chem. C, 2007, 111, 9811-9817.

44 F. Zuo, K. Bozhilov, R. J. Dillon, L. Wang, P. Smith, X. Zhao, C. Bardeen and P. Feng, Angew. Chem., Int. Ed., 2012, 51, 6223-6330.

45 B. Chen, J. Qiu, E. Sakai, N. Kanazawa, R. Liang and H. Feng, ACS Appl. Mater. Interfaces, 2016, 8, 17659-17667.

46 Y. Lu, S. Sathasivam, J. L. Song, C. R. Crick, C. J. Carmalt and I. P. Parkin, Science, 2015, 347, 1132-1135. 